\title{
Announcement: Change of Editor
}

Behavioural Pharmacology 2020, 31:1-2

After 30 years at the helm of Behavioural Pharmacology, and a personal involvement in the review of over 2000 manuscripts, I stepped down as Chief Editor at the end of 2019. It has been a huge pleasure and privilege to occupy a role that has provided a bird's eye view of the maturation of behavioural pharmacology into a discipline characterized by the expert use of novel neuroscience methodologies against a background of sound and sophisticated behavioural designs. It has also been my privilege, in my 'rest of world' field editor role, to provide support to scientists from less developed and non-English-speaking countries, including helping them to turn good science into readable prose.

I am delighted also to announce that Louk Vanderschuren has stepped up from his current role as Associate Editor to take over as Chief Editor, and I wish Louk every success in his new and demanding role. (We have essentially swapped roles, as I will be staying on for a while as Associate Editor to support Louk through the transition.) I also take the opportunity to express my thanks and appreciation for their sterling and constant support to Louk and our third Editorial colleague, Jack Bergman (and their predecessors as Associate Editor, David Sanger, Michael Oglesby and Alice Young); as well as our Reviews Editorial team of Bart Ellenbroek, Gernot Riedel, Viviana Trezza and Ben Yee; and all of the present and past members of our Editorial Board, too numerous to mention by name. A final word of thanks goes to the multitude of scientists who have entrusted us with their work to evaluate. I hope they have been largely satisfied with the outcomes.

Paul Willner January 2020 
It is an honour to follow in the footsteps of Paul Willner as Chief Editor of Behavioural Pharmacology. For the last 10 years, I have enjoyed the pleasant and constructive collaboration with Paul, Jack Bergman and our Reviews Editors team in managing the scientific content of this journal and I look forward to the continuation of this process. Demanding as the role of Chief Editor may be, I see it as a positive challenge to lead Behavioural Pharmacology into the new decade.

Since the turn of the century, the general view on the position of pharmacology in the scientific landscape has changed, perhaps because the development of new pharmacotherapies has not yielded many spectacular breakthroughs. At the same time, pharmacological methods have remained a trusted means to investigate human and animal physiology and behaviour, while the introduction of novel methodologies such as chemogenetics and optogenetics have provided new dimensions to using pharmacology to understand behaviour. These days, many scientists no longer rely on single approaches and methodologies in their studies, which has led to fascinating research endeavours in which pharmacology, psychology, psychiatry and neuroscience are combined, to employ their respective strengths. I therefore see many opportunities for Behavioural Pharmacology to be the journal for the publication of important discoveries in our understanding of the relationship between brain function and behaviour, be they of fundamental or applied, and of mono- or multidisciplinary nature.

Let me end by expressing my gratitude and appreciation to Paul Willner for being the Chief Editor of Behavioural Pharmacology for so many years. With his unflagging effort, enthusiasm and support - for authors, reviewers and editors - he has set a strong example of how to manage an international, peer-reviewed scientific journal, in a way that deserves profound admiration.

Louk Vanderschuren January 2020 